\title{
Diagnosis and Treatment of Laparoscopic Cholecystocolonic Fistula and Cholestoenteric Fistula
}

\author{
Mubarak Ali Kirih, Zheng Junhao, Cai Jingwei, Xie Yangyang, Faisa Ali Abdi, Tao Liye and Xiao Liang* \\ Department of General Surgery, Sir Run Run Shaw Hospital, College of Medicine, Zhejiang University, Hangzhou, Zhejiang \\ Province, China
}

"Corresponding author: Xiao Liang, Department of General Surgery, Sir Run Run Shaw Hospital, College of Medicine, Institute of Minimally Invasive Surgery, Zhejiang University, No. 3 East Qingchun Road, Hangzhou, Zhejiang Province, China, Tel: 008613588708506; Email: srrshlx @zju.edu.cn

\footnotetext{
Abstract

Combined with a specific case, CT diagnosis of biliary-intestinal fistula with gallstone intestinal obstruction was analyzed.

It was concluded that abdominal plain film was used to diagnose gallstone intestinal obstruction.

The key is to observe whether there are positive stones and pneumogallstone in the intestine. The specific imaging features can be obtained by CT diagnosis of cholecystoenteric fistula and surgical treatment of laparoscopic cholecystocolonic fistula.
}

Keywords: Cholelithiasis; Gallstone ileus; Cholecystocolonic fistula

Received Date: October 27, 2019; Accepted Date: November 11, 2019; Published Date: November 18, 2019

\section{Case Report}

A 40-year-old man presented to the emergency department complaining of right upper abdominal pain for 10 days, with no obvious cause of the emergence of right upper abdominal pain and distension, no obvious change of position improved, no fever chills, nausea, and discomfort, there was no bowel movement or stool vital signs were within normal limits and admit examination: body temperature - $36.2^{\circ} \mathrm{c}$, pulse - 86 times $/ \mathrm{min}$, breath - 20 times $/ \mathrm{min}$, blood pressure - 100/90 $\mathrm{mmHg}(1 \mathrm{mmHg}$ $0.133 \mathrm{kPa}$ ). CT scan showed a hint of gallbladder duodenal fistula, gallbladder pneumatosis, cholecystitis, and right middle intestine oval laminated gallstone size was $4 \mathrm{~cm}$ with proximal segment obstruction, distal intestinal obstruction catheter located in the near side Small calcification in the wall of the aorta. We gave anti-inflammatory and other symptomatic relief treatment and there was no significant improvement. Check on the abdominal CT scan showed as acute intestinal obstruction, cholecystitis, and duodenal leakage. "The patient was sent for further diagnosis and treatment, an emergency department, to check on the abdomen and pelvis. We improved gallbladder duodenal fistula, prompt gallbladder pneumatosis, cholecystitis,

Citation: Mubarak Ali Kirih, Diagnosis and Treatment of Laparoscopic Cholecystocolonic Fistula and Cholestoenteric Fistula. J Clin Cases Rep 3(3): 115-122. 
right middle intestine oval laminated gallstone size was $4 \mathrm{~cm}$ with proximal segment obstruction, distal intestinal obstruction catheter located in the near side". The cause of obstruction was found to be large stones in the ascending colon diverticulum. Small calcification in the wall right middle intestine oval laminated gallstone size was $4 \mathrm{~cm}$ with proximal segment obstruction. There was no special discomfort and no treatment. Since the disease, the spirit, no obvious weight loss. Laboratory and special examinations of liver, gallbladder, pancreas, and spleen ultrasound was performed. Liver, gallbladder, pancreas, spleen ultrasound examination: emergency, the size of the liver was normal, the capsule was smooth, the echo of the liver was enhanced, the vascular network was clear, no obvious occupying was found, and there was no obvious expansion of the intrahepatic bile ducts. The size and shape of normal gallbladder wall thickening was rough and about $0.56 \mathrm{~cm}$ thick, cavity bile perforated poor, with acoustic shadow visible hyper echo multiple, larger size of about $0.86 \mathrm{~cm}$. There was no obvious widening of the inner diameter of the upper segment of the common bile duct, which did not show obvious abnormality in the segment of the segment. The shape of the spleen was normal and the echo in the spleen was even and fine. There was no obvious abnormal appearance of the pancreas, uniform internal echo, no obvious expansion of the main the pancreas, duct and no significant lesion Figure 1 - Figure 4.
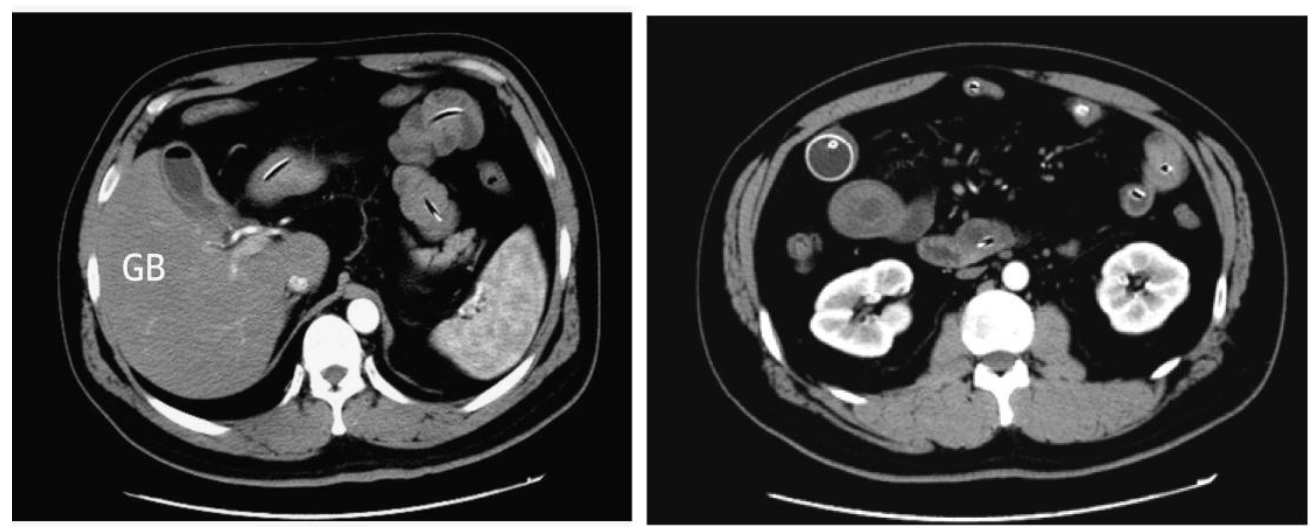

Figure 1: This is the Computed abdominal tomography.

A hint of gallbladder duodenal fistula, gallbladder pneumatosis, cholecystitis, right middle intestine oval laminated gallstone size was $4 \mathrm{~cm}$ with proximal segment obstruction, distal intestinal obstruction catheter located in the CT scan showed as acute intestinal obstruction, cholecystitis, duodenal leakage". The patient was further diagnosed and treated by an emergency department to check on the abdomen and pelvis.

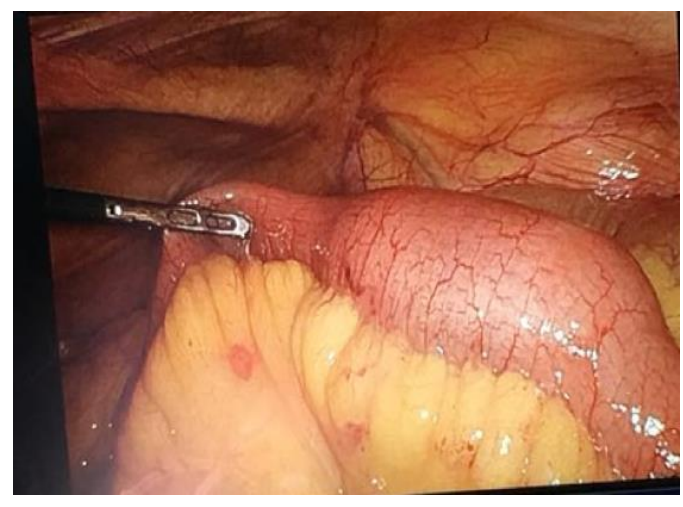

Figure 2: The stone long diameter of about $4 \mathrm{~cm}$ with the proximal segment obstruction, distal intestinal obstruction catheter located in the near side of duodenum and small ascending colon diverticulum. 
We improved gallbladder duodenal fistula, prompt gallbladder pneumatosis, cholecystitis, right middle intestine oval laminated gallstone size was $4 \mathrm{~cm}$ with proximal segment obstruction, distal intestinal obstruction catheter located in the near side." The cause of obstruction was found to be large stones in the ascending colon diverticulum. Small calcification in the wall right middle intestine oval laminated gallstone size was $4 \mathrm{~cm}$ with proximal segment obstruction.
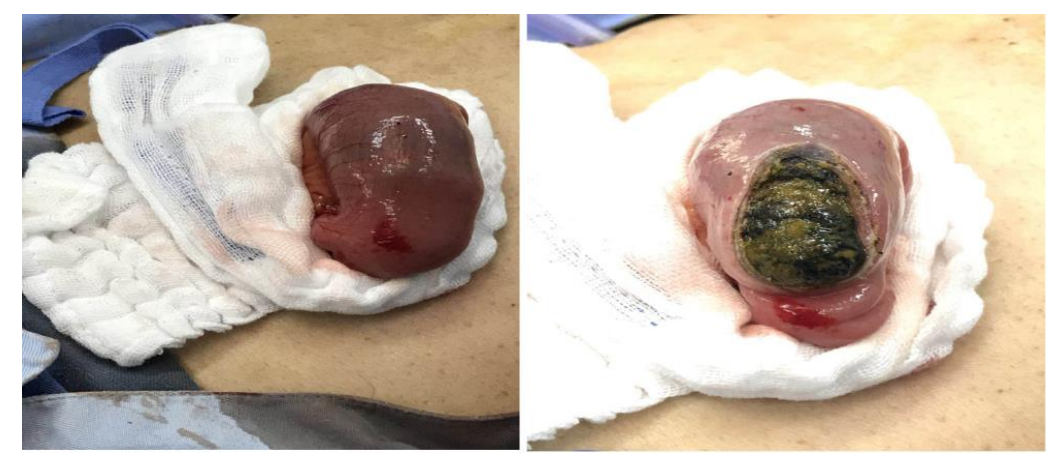

Figure 3: During exploration, (A) trace of the stone in the intestinal loops and (B) the resected intestinal segment and the stone the patient was found to have the duodenal obstruction.

\section{In operation}

The largest gallstone shadow disappeared in the gallbladder, the gallbladder was pneumatous, and the boundary between gallbladder and duodenum was not clear.
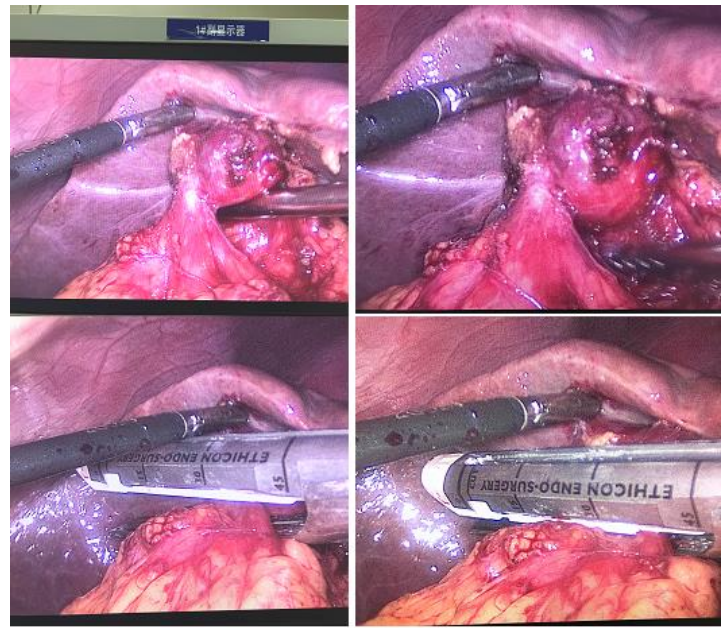

Figure 3: Duodenum indrawn to the fundus of gallbladder with dense adhesions on laparoscopy.

The Diagnostic laparoscopy was done which revealed dense omental adhesions and fat stranding covering the Gallbladder fossa and inferior surface of liver as shown in picture. Adhesiolysis was done duodenum was seen in close proximity to liver and adherent to it. A window was created between gall bladder, duodenum and the liver isolating the fistulous communication to gall bladder.

\section{Discussion}

\section{Incidence and etiology of gallbladder-colon fistula}

The incidence of gallbladder-duodenal fistula was the highest i.e. approximately 1\% 8\% [1]. Gallbladder-colon fistula was the second and gallbladder-stomach fistula was the least [2]. Gallbladder colon fistula clinically, it is very rare, accounting 
http://www.tridhascholars.org | July-2020

for only $8 \%$ [3] of bilio intestinal fistula. More than $90 \%$ of the causes of gallbladder-colon fistula are gallstones. Other rare causes include gastrointestinal ulcer, biliary tract or intestinal swelling Tumors, tuberculosis, colonic inflammatory lesions, etc. When B-mode ultrasonography revealed ampullary space-occupying and intrahepatic and extrahepatic bile ducts Expansion, gallbladder atrophy, adhesion with surrounding tissues, unclear outline by domestic research [4], if clinical nonobstructive jaundice often reflects pathological biliary fistula. Yes, no tumors or intestinal diseases were found as well as Stones, etc. The cause of the disease is that the inflammation between the biliary tract and intestine recurs and adheres closely. Gallstones in the gallbladder affect bile excretion and cause congestion and edema in the gallbladder wall. Incarceration of gallstones may occur when the gallstones are large and the gallbladder wall is repeated due to the gallstones. Friction or stone compression causes ischemia of gallbladder wall and perforation after gangrene. Perforation and biliary tract also occur after the intestinal tract, which is tightly adhering to the gallbladder, is involved for inter-abnormal channel formation [5]. Compression of the bottom of gallbladder leads to poor blood supply, which is liable to adhere to the transverse colon and form chronic internal fistula. Cholecystoduodenal fistulas are mostly located between the neck of the gallbladder and the duodenal bulb.

No bilirubin-hepatic circulation abnormalities occur when duodenum was discharged. Therefore, these patients had no diarrhea and poor absorption of vitamin $\mathrm{K}$. This is the biggest difference between the both gallbladder-colon fistula and gallbladder-duodenum.

The pathogenesis of intestinal fistula is similar, but the pathophysiological changes are different. The different location also determines the different treatment methods of the two groups.

\section{Pathophysiological changes of gallbladder and colonic fistula and clinical correlation}

Normally, intestinal gas cannot enter the biliary tract. Gas from the intestine enters the biliary tract after the formation of intestinal fistula.

Pneumoconiosis is an important basis for the preliminary diagnosis of Bilio intestinal fistula. According to report stones may be drained through the sinus between the biliary and intestinal tracts after the formation of internal biliary and intestinal fistula Up to the intestinal tract, bile is drained after the pressure of the biliary tract is reduced. Biliary inflammation temporarily improved, but intestinal contents can also reflux to the gallbladder. The biliary tract infection is aggravated by biliary tract infection. The etiology of repeated biliary tract infections may occur repeatedly in patients with biliary tract infections show symptoms and signs of infection. When the biliary-intestinal passage is depressed due to intragallbladder pressure. Bile is obstructed by low fistula size and granulation tissue growth. Lack of drainage aggravates gallbladder and biliary tract infections [6].

After the formation of biliary-intestinal fistula, bile is directly discharged into the intestinal tract with low pressure through the fistula orifice. The pressure of gallbladder and biliary tract is reduced. Gallbladder atrophy occurs in most patients with biliary-intestinal fistula [7]. After gallbladder-colon fistula, bile enters the colon directly and cannot maintain normal bilirubinliver circulation.

Decreased absorption of fat-soluble vitamins such as vitamin K can lead to coagulation dysfunction, while increased intracolon bile salts directly stimulate the colon, patients may have diarrhea [8] as per some scholars [9]. 
http://www.tridhascholars.org | July-2020

Chronic diarrhea, biliary tract pneumoconiosis and vitamin $\mathrm{K}$ malabsorption are the specific triple characteristics of gallbladder-colon fistula. After gallbladder-duodenal fistula occurs, bile is directly discharged into the duodenum without abnormal bilirubin-liver circulation. Therefore, these patients had no diarrhea and poor absorption of vitamin K. This is the biggest difference between the two gallbladder-colon fistula and gallbladder-duodenum. The pathogenesis of intestinal fistula is similar, but the pathophysiological changes are different. Different locations also determine the difference between the two treatments.

\section{Clinical manifestations and diagnosis of cholecystocolonic fistula}

Cholecysto-intestinal fistula patients usually have no specific clinical manifestations. Because of the larger age of patients with gallbladder and colonic fistula, they often suffer from chronic diseases such as cardiovascular disease and diabetes. The abdominal pain in patients is often covered by cholelithiasis and gallbladder inflammation. Gastrointestinal ulcer can also appear in the cap as some scholars recognize [9].

For gallbladder-colon fistula patients with a long history of biliary irritation nodules Diarrhea in the intestine is the key to the diagnosis of the disease. Gallbladder-colon fistula acute episodes often present as gallstone intestinal obstruction and now, the digestive tract bleeding and other abnormalities were observed. Acute gallbladder-duodenal fistula chole lithic ileus may also occur, but most of the ileus is small intestine considered as obstruction [10].

For patients with a long history of biliary calculi, the onset of the disease is early. It is characterized by acute inflammation of biliary tract such as abdominal pain, chills, fever and jaundice. Then symptoms suddenly relieve or appear gastrointestinal bleeding, obstruction, etc. Now patients should consider the possibility of cholecystocolonic fistula. All kinds of imaging examinations are lack of specificity for gallbladder-colon fistula. Combined use of multiple auxiliary examinations can improve preoperative gallbladder-colon fistula and fistula diagnosis rate. Abdominal ultrasonography is the most commonly used method of examination. But abdominal ultrasonography can only detect gallbladder atrophy and biliary tract volume. Indirect signs of gallbladder-colon fistula such as disappearance of dark bile area in gas and gallbladder elephants cannot directly display [11].

Spiral CT can detect biliary gas accumulation, inflammatory tissue thickening around gallbladder and intestinal anomalies. Indirect signs such as normal low-density shadow dilated intestinal canal and contrast medium shunt can also display the situation of gallbladder-colon fistula from multiple angles, comprehensively and stereoscopically through three-dimensional reconstruction. Spiral CT examination is the main auxiliary examination of gallbladder-colon fistula [12].

Some scholars think that spiral CT hypotonic enhanced scan is more practical. The tissue of gallbladder wall, intestinal wall and sinus wall thickened with chronic inflammation is enhanced when the tension of gallbladder and intestine cavity decreases. After that, the gas in the sinus tract can be better displayed, and thus the gas in the sinus tract can be clearly displayed. It shows the ruptured gallbladder wall, so the presence of fistula can be identified [13-15].

MRI can more accurately display negative stones such as cholesterol stones. But the requirement of MRI for patients is higher and the scanning time is longer. The display of fistula is not as good as that of spiral CT, which can be used as a supplement to spiral CT. 
http://www.tridhascholars.org | July-2020

Biliary tract pneumoconiosis was found by preoperative imaging examination. The diagnosis of biliary tract pneumobarium double contrast radiography should be further confirmed. Among the 2 cases diagnosed before operation, 1 case underwent preoperative diagnosis.

CT examination revealed biliary tract pneumoconiosis, and ERCP examination was performed to confirm the diagnosis.

X-ray examination of barium enema is the most effective method for diagnosis of biliary-intestinal fistula. It can not only determine the location of fistula, but also show the fistula. The size and location of the fistula, but the positive rate of the examination was affected by the size of the fistula. The limitation of this is granulation tissue blockage and patient's body position. Digestion Endoscopy can directly show the location and size of fistula. It can intuitively understand the situation of fistula, and it is easy to operate and complications less and we should use a lot of this [16]. The authors believe that ERCP is useful in the diagnosis of biliary and intestinal diseases. ERCP can clearly understand the sinus tract, the type of internal fistula, and directly observe the distal bile duct for correlation of therapeutic operation [17-18].

However, due to the invasive examination, it should be done clinically. Strictly grasp the indications and contraindications of abdominal B-mode ultrasonography and upper gastrointestinal tract construction, combination of shadow enema, barium enema, CT, MRCP and ERCP. The application can not only determine the location of fistula, but also find the cause of fistula on basis for supply.

\section{Treatment of cholecystocolonic fistula}

The treatment of gallbladder-colon fistula is mainly surgery, but it cannot be cured without surgery. The key to the operation is to remove the biliary-intestinal fistula and repair the intestinal fistula. Normal biliary drainage was restored. The principle of treatment is to remove gallbladder and stone. Cutting off fistula and repairing intestinal fistula were the main methods [19].

Choosing appropriate surgical methods and supportive treatment after operation can effectively reduce the incidence of complications.

The occurrence of complications and the adhesion around gallbladder should be carefully separated during operation. To identify the anatomy and understand the sinus condition there is lack of clarity on the tissue level and gallbladder atrophy which make cholecystectomy more and more important.

Difficulty, the gallbladder can be separated from the bottom of the gallbladder, and then followed up. If the common bile duct is found in the cystic duct, the gallbladder need not be resected completely. Partial cholecystectomy may be performed if necessary. Resection of scar tissue in colon fistula after the operation, double-layer, intermittent and double-layer colon fistula can be used for the patients with better colon fistula.

Stage I suture repair of colon fistula by varus suture; colon fistula Poor oral condition, such as large fistula, marked edema of tissue, intestinal tract Inadequate preparation, high tension after suture and fistula repair after suture If the risk of intestinal fistula is high, the omentum coverage should be increased after suturing the fistula. Risk of intestinal fistula after anastomosis due to severe local inflammation and poor blood supply. 
http://www.tridhascholars.org | July-2020

Partial bowel resection is feasible for the larger patients, and the general condition of the patients is poor. If long-term operation is not tolerated and intestinal infection is serious, it may be concluded with enterostomy [20].

At present, it is considered that cholecystocolonic fistula is not a laparoscopic hand, it is operative contraindications [1]. After careful separation of adhesion, a thinner fistula can be obtained [21]. It was directly clipped by ligation or titanium clips.

Most fistulas can be sutured directly, continuously or intermittently, and adhere to the fistula near the anatomical separation. When it is organized, it must not be separated from each other. It is an important method because its damage range often exceeds the visual vision [22]. Fistula week peri scar tissue should be routinely examined rapidly during operation to exclude gallbladder or the possibility of intestinal malignant tumors [23]. Some studies [24] argue that, for the sake of reducing the incidence of biliary tract injury and avoiding peripheral organs as much as possible. Iatrogenic injury, decisive conversion to laparotomy is still the best choice of treatment.

Preoperative diagnosis of cholecystocolonic fistula should be considered in patients with intestinal preparation, preoperative intestinal preparation can reduce abdominal sensation in patients with cholecystocolonic fistula. The risk of infection and anastomotic fistula should be paid special attention to during operation and incision contamination [25].

\section{Conclusion}

Due to the existence of abnormal channels between biliary tract and intestinal tract, the patients repeatedly suffer from abdominal pain, jaundice, high fever and other symptoms and signs of biliary tract infection, which cannot be cured without surgery, and are highly suspicious before operation. Gallbladder-colon fistula can be improved by multiple examinations in patients with gallbladder-colon fistula.

Preoperative diagnosis rate and preoperative diagnosis are very important for the complete treatment and prognosis of gallbladder-colon fistula.

\section{References}

1. Angrisani L, Corcione F, Tartaglia A, et al. (2001) Cholecystoenteric fistula (CF) is not a contraindication for laparoscopic surgery. Surgical Endoscopy 15(9): 1038-1041.

2. Huang BK, Chess MA (2009) Cholecystitis of a duplicated gallbladder complicated by a cholecystoenteric fistula. Pediatric Radiology 39(4): 385-388.

3. Yamashita H, Chijiiwa K, Ogawa Y, et al. (1997) The internal biliary fistula-reappraisal of incidence, type, diagnosis and management of 33 consecutive cases. Hepato-Pancreato-Biliary (HPB) Surgery 10(3): 143-147.

4. Yang YG, Li MY, Dai D, et al (2008) Ampullary carcinoma with pathologic Cholecysto-intestinal fistula: A report of 12 cases. Chinese Journal of General Surgery 17(3): 253-255.

5. Tahir OM, Fedele CR, Sneider MB, et al. (2014) Cholecystocolonic fistula. Delaware Medical Journal 86(12): 373-375.

6. Song BJ (2014) Progress in diagnosis and treatment of gallbladder intestinal fistula. Chinese Journal of Surgery of Integrated Traditional and Western Medicine 20(5): 566-568.

7. Liu YF, Zhang L, Li XB, et al. (2015) Experience in diagnosis of biliary fistula in 21 cases. Chinese Journal of Current Advances in General Surgery 18(3): 243-244. 
8. Savvidou S, Goulis J, Gantzarou A, et al. (2009) Pneumobilia, chronic diarrhea, vitamin K malabsorption: A pathognomonic triad for cholecystocolonic fistulas. World Journal of Gastroenterology: WJG 15(32): 4077-4082.

9. Costi R, Randone B, Violi V, et al. (2009) Cholecystocolonic fistula: Facts and myths. A review of the 231 published cases. Journal of Hepato-Biliary-Pancreatic Surgery 16(1): 8-18.

10. Engelberger S, Schuld J, Schilling MK, et al. (2010) Cholecystocolonic fistula prevents upper intestinal obstruction by a large gallstone after perforation into the duodenum. Langenbeck's Archives of Surgery 395(1): 95-97.

11. Zhu WJ, Xu YZ, Zhou ZL, et al. (2006) Ultrasonographic features and misdiagnosis of gallbladder duodenal fistula. The Journal of Practical Medicine 18: 2164-2165.

12. Carlsson T, Gandhi S (2015) Gallstone ileus of the sigmoid colon: an extremely rare cause of large bowel obstruction detected by multiplanar CT. BMJ case reports 2015.

13. Singh AK, Gervais D, Mueller P (2004) Cholecystocolonic fistula: Serial CT imaging features. Emergency Radiology 10(6): 301-302.

14. He B, Gong HH, Chen YY, et al. (2007) The value of spiral CT hypotonic enhanced scan in diagnosing cholecystoduodenal fistula. Practical Clinical Medicine 8(1): 100-102.

15. Chick JFB, Chauhan NR, Paulson VA, et al. (2013) Cholecystocolonic fistula mimicking acute cholecystitis diagnosed unequivocally by computed tomography. Emergency Radiology 20(6): 569-572.

16. Engelberger S, Schuld J, Schilling MK, et al. (2010) Cholecystocolonic fistula prevents upper intestinal obstruction by a large gallstone after perforation into the duodenum. Langenbeck's Archives of Surgery 395(1): 95-97.

17. Yang XW, Jiang Y, Qin XH, et al. (2010) Experience in diagnosis and treatment of 23 cases of biliary fistula. Journal of Hepatopancreatobiliary Surgery 22(2): 123-124.

18. Rice JP, Spier BJ, Soni A (2010) Preoperative diagnosis of cholecystocolonic fistula on ERCP. The New Zealand Medical Journal 123(1311): 69-72.

19. Verhoeven Y, Bac DJ (2011) Cholecystocolonic fistula. Clinical Gastroenterology and Hepatology 9(10): A30.

20. Balent E, Plackett TP, Lin-Hurtubise K (2012) Cholecystocolonic fistula. Hawaii Journal of Medicine \& Public Health 71(6): 155-157.

21. Godquin B, Favre R, Blanchard J, et al. (1974) A cholecysto-colic fistula caused by perforation of a gallbladder cancer. Case report (author's transl). Acta Gastro-enterologica Belgica 37(4): 236-242.

22. Liu YL, Ding YM, Wang CT, et al. (2008) Diagnosis and treatment of internal biliary fistula (A report of 48 cases). Journal of Clinical Surgery 16(10): 668-669.

23. Chowbey PK, Bandyopadhyay SK, Sharma A, et al. (2006) Laparoscopic management of cholecystoenteric fistulas. Journal of Laparoendoscopic \& Advanced Surgical Techniques 16(5): 467-472.

24. Liu T, Chen LH (2010) Clinical analysis of gallbladder colon fistula complicated with gallbladder stone. Medical Journal of West China 22(2): 288-289.

25. Gelbman A (2006) Gallstone ileus with cholecystocolonic fistula. Emergency Radiology 12(4): 199-200. 